\title{
COVID-19 Vaccine Intent Among Health Care Professionals of Queen Elizabeth Hospital, Barbados
}

\author{
Kandamaran Krishnamurthy (D) \\ Natasha Sobers ${ }^{2}$ \\ Alok Kumar iD ' \\ Nkemcho Ojeh (iD) \\ Andrea Scott ${ }^{3}$ \\ Clyde Cave ${ }^{3}$ \\ Subir Gupta' \\ Joanne Bradford-King ${ }^{3}$ \\ Bidyadhar Sa (iD) ${ }^{4}$ \\ Oswald Peter Adams (iD) \\ Michael H Campbell (D) \\ Md Anwarul Azim Majumder (D) \\ 'Faculty of Medical Sciences, The \\ University of the West Indies, Cave Hill \\ Campus, Bridgetown, Barbados; \\ ${ }^{2}$ Caribbean Institute for Health Research, \\ The University of the West Indies, Cave \\ Hill Campus, Bridgetown, Barbados; ${ }^{3}$ The \\ Queen Elizabeth Hospital, Bridgetown, \\ Barbados; ${ }^{4}$ Faculty of Medical Sciences, \\ The University of the West Indies, St \\ Augustine, Trinidad and Tobago
}

Introduction: Due to increased exposure risk and the potential impact of COVID-19 infection, health care professionals (HCP) are a target group for COVID-19 vaccination. This study aimed to examine the acceptability of COVID-19 vaccines among HCP at the Queen Elizabeth Hospital, Barbados.

Design and Methods: A cross-sectional survey of HCP was conducted between February 14 and 27, 2021 using an online questionnaire. The questionnaire included demographic information, knowledge of novel coronavirus, intention to accept the COVID-19 vaccination, vaccine literacy (VL), and perceptions and attitudes regarding COVID-19 vaccines. Mean VL scores were calculated. The relationship between socio-demographic variables and vaccine intent was assessed using a multivariable logistic regression model. Results: Of $343 \mathrm{HCPs}, 55.1 \%$ indicated they would accept the COVID-19 vaccine if it were available; $44.9 \%$ expressed hesitancy towards the COVID-19 vaccine. We assessed the impact of socio-demographic factors and previous vaccine behavior on vaccine intent; after adjustment of the multivariable logistic regression model, non-Barbadian nationality and previous flu vaccine uptake were statistically significant predictors of reported intent to take the COVID-19 vaccine. Persons who indicated that they would take the vaccine had a higher mean vaccine literacy score $[3.4695 \%$ CI $(3.40,3.52)]$ than those who were not ready to take the vaccine immediately $[3.2395 \%$ CI $(3.15,3.30)]$. VL scores were higher among the $29.5 \%$ of HCPs who believed vaccines should be mandatory.

Conclusion: This study highlighted vaccine hesitancy among HCPs in the sole public tertiary hospital of Barbados. As HCP perceptions may help or hinder the campaign to promote vaccine uptake in Barbados, vaccine promotion programs targeting HCPs are needed to ensure the success of the country's COVID-19 vaccination drive.

Keywords: vaccine intake, vaccine hesitancy, vaccine literacy, healthcare professionals, COVID-19, Barbados

\section{Introduction}

The development of effective COVID-19 vaccines is a remarkable scientific achievement that occurred with unprecedented speed. The alarming scope of COVID-19 mortality and morbidity led drug regulatory authorities to authorize emergency use of some vaccines from the early stages of the pandemic. ${ }^{1-3}$ Historically, vaccination has been the most effective control for many infectious diseases, preventing millions of deaths and disabilities. Notwithstanding efforts to curb the COVID-19 pandemic by preventive public health measures such as
Correspondence: Md Anwarul Azim Majumder

Faculty of Medical Sciences, The

University of the West Indies, Cave Hill

Campus, Bridgetown, Barbados

Email azim.majumder@cavehill.uwi.edu 
wearing masks, sanitizing hands regularly, maintaining a safe distance, avoiding crowded indoor areas, and spreading awareness among the general public, effective and safe vaccination has emerged as currently essential to mitigate disease and death. ${ }^{4}$

Fast-tracked COVID-19 vaccine development and regulatory approval have contributed to skepticism among potential vaccine recipients. Further, the spread of misinformation and prominence of unreliable sources have fueled doubt and worry that politics, rather than science, may be the driving force in vaccine development. ${ }^{5}$ COVID-19 vaccine acceptance is also influenced by social media, contributing to a phenomenon the World Health Organization (WHO) has characterized as an 'infodemic' of misinformation and rumors that make it difficult to identify truly scientific and reliable sources of information. ${ }^{6}$

Vaccine hesitancy among the general population has presented challenges to public health response even prior to the COVID-19 pandemic, and vaccine refusal has led to the outbreak of other infectious diseases. ${ }^{7}$ Historical examples show that vaccine boycotts can result in a resurgence of the disease. Inadequate (and politicized) public health response has triggered concerns that vaccines alone may not be sufficient to develop herd immunity against SARSCoV-2. ${ }^{8}$ These views are reflected in recent surveys revealing widespread uncertainty regarding acceptance and efficacy of COVID-19 vaccines. ${ }^{9,10}$ A systematic review of 31 studies from 33 countries reported vaccine acceptance rates ranging from $23.6 \%$ (Kuwait) to $97 \%$ (Ecuador) among the general population and from 27.7\% (Democratic Republic of the Congo) to $78.1 \%$ (Israel) among healthcare workers (8 studies, including doctors and nurses). ${ }^{11}$ A study conducted by the Pan American Health Organization (PAHO) in the Caribbean region reported that $77 \%$ of the healthcare workers indicated they would receive a COVID-19 vaccine as soon as possible. ${ }^{12}$ The contributing factors to hesitancy include negative perception of vaccine efficacy, safety, convenience, and price. ${ }^{13}$ Vaccination behavior of the general population is influenced significantly by both professional recommendations and personal behavior of HCPs. ${ }^{14}$ Widespread refusal or hesitancy among HCPs influences public trust, attitudes, intentions, and beliefs critical for the success of vaccination programs. ${ }^{8,15,16}$ Vaccine acceptance rates below $60 \%$ could be the major hindrance to preventing infection and slowing transmission of COVID-19. ${ }^{11}$
The first COVID-19 case was identified in Barbados on 17 March 2020. Due to surges in COVID-19 cases, Barbados has strengthened public health measures at various points in the pandemic. As of Nov 1, 2021, 6.2\% of the Barbadian population was tested positive for COVID19 , and the death rate was 0.53 per 1000 people. ${ }^{17}$ There have been more than 18,000 confirmed cases and 156 deaths (Nov 1, 2021). ${ }^{18}$ The Barbados Ministry of Health and Wellness began offering vaccines at QEH and other public primary care facilities in February 2021, and by 1 November 2021, 47\% of the population had been fully vaccinated in Barbados. ${ }^{19}$ Due to the increased risk of exposure and the potential impact of COVID-19 infection among medical personnel, HCPs are priority target populations for COVID-19 vaccines in many countries, including Barbados.

Against this background, the present study sought to identify perceptions and attitudes related to COVID-19 vaccine acceptance among HCPs of the Queen Elizabeth Hospital (QEH), Barbados.

\section{Methods}

\section{Setting}

The Queen Elizabeth Hospital (QEH), situated in the capital city of Bridgetown, is the main general hospital in Barbados. It opened in November 1964 and is an accredited teaching hospital affiliated with the University of the West Indies, Cave Hill Campus for health professional training. With a bed capacity of 600, QEH employs approximately 850 health care professional staff and boasts various medical, surgical, and other departments, including Accident and Emergency.

\section{Ethical Approval}

Ethical approval was obtained from the University of the West Indies, Cave Hill Campus/Barbados Ministry of Health and Wellness Research Ethics Committee/ Institutional Review Board (IRB No. 210101-B) and the QEH Ethics Committee (Ref: 12021) and the study was conducted in accordance with the guidelines of the 1975 Declaration of Helsinki.

\section{Study Design, Sampling and Data Collection}

The study used purposive sampling of all HCPs working at the QEH, who were invited to complete a cross-sectional online survey assessing perceptions and attitudes regarding 
COVID-19 vaccination. Inclusion criteria were all HCPs ( $\mathrm{n}=850$ ), including doctors, nurses, pharmacists, physiotherapists, radiographers, laboratory technologists/technicians, and others currently employed at the QEH. Email invitations were sent during the period of February 14 to 27, 2021 with a Google Forms link to the survey.

Questionnaire development was informed by a literature review conducted by one of the authors (MAAM). The questionnaire was pretested, and final version was reviewed and approved by the research team. Items were grouped in the following sections: (A) demographic information, (B) knowledge of novel coronavirus (COVID-19), ${ }^{20}$ (C) current flu and other vaccine-related behaviour, ${ }^{20,21}$ (D) acceptance of COVID-19 vaccination, ${ }^{8,20}$ (E) vaccine literacy interactive/critical skills, ${ }^{20,22}$ (F) COVID-19 vaccines perceptions and attitudes, ${ }^{20,21}$ and $(\mathrm{G})$ Further comments (Appendix 1). No identifying information was collected.

Vaccine literacy (VL) levels were assessed by adapting questions from a self-report questionnaire for adulthood vaccination. $^{22,23}$ We used six items that evaluated interactive-critical VL which focused on cognitive efforts (eg, problem-solving, decision-making), following the definition proposed by Nutbeam. ${ }^{24}$ These six items were as follows: (1) Have you consulted more than one source of information? (2) Have you got the information you were looking for? (3) Have you had the opportunity to use the information? (4) Have you discussed your knowledge of vaccinations with your colleagues? (5) Have you considered the credibility of the sources? (6) Have you found any useful information to decide for vaccination? We calculated Cronbach $\alpha$ for these items to assess the internal consistency of the adaptation.

\section{Statistical Methods}

We calculated mean age and proportions for gender, marital status, and nationality. We utilized the PROGRESS+ framework to choose social determinants to explore associations between socio-demographic variables and the main outcome variable: intent to take COVID-19 vaccine. The socio-demographic variables measured and fit into the framework were: Barbadian nationality (place); occupation, gender, marital status (a proxy for social capital); and age (measured as a categorical variable). Variables were categorized in the following manner: Nationality Barbadian or non-Barbadian nationality; Occupation nurses, allied health/administration and doctors; Gendermale or female; Marital status - married or unmarried (including divorced, never married, and widowed). Age was categorized as $18-34$ and 35 plus.

Vaccine intent was measured as participants' indication of their intention to become vaccinated by completing the statement, "If a new COVID-19 vaccine becomes publicly available I intend/I do not intend ...." The options were as follows: (1) I intend to get it as soon as possible; (2) I intend to wait to see how it affects others before I get it; (3) I do not intend on getting it soon, but might sometime in the future; (4) I do not intend to ever get the vaccine. Persons who indicated that they intended to take the vaccine but would wait until others get it first or who stated that they do not intend to take the vaccine now or that they do not intend to take the vaccine ever were classified as "vaccine-hesitant." Perceived knowledge was measured by asking, "How would you rate your knowledge level on COVID-19?"

The relationship of sociodemographic variables with vaccine intent was assessed using chi-squared tests to determine unadjusted association. Adjusted associations were assessed using a multivariable logistic regression model examining the associations between perceived knowledge, the selected sociodemographic factors, and vaccine intent. We determined the adjusted relationships between socio-demographic factors, previous behavior (acceptance of seasonal influenza vaccine) perceived knowledge, and the main outcome variable - vaccine intent. Missing data for eight cases were handled using the zero imputation method for the vaccine intent variable. Given the small numbers of persons who rated their knowledge as poor, the knowledge variable was dichotomized as "average or poor" versus "good".

In building the multivariable logistic regression model, we used a hierarchical approach by entering the PROGRESS + social determinants as predictors of vaccine intent into the initial model (Model 1). We then added perceived knowledge and previous vaccination behavior to determine if these improved model predictions (Model 2). Both models showed good fit as measured by the Hosmer-Lemeshow test: Model 1 p-value $=0.86$; Model 2 p-value $=0.54$. We compared model fit using the likelihood ratio test and found the fit of Model 2, which correctly classified $65 \%$ of observations, to be significantly higher than that of Model 1, which correctly classified $62 \%$ of observations.

Vaccine literacy skills were explored using the six-item scale described above. The mean and median values for VL skills were calculated for the socio-demographic 
factors (Barbadian nationality, occupation, gender, marital status (a proxy for social capital), and age (dichotomized into less than and over 35 years). The correlations between COVID-19 perceptions and beliefs and vaccine literacy levels were assessed using Spearman's rank correlation tests, given the ordinal nature of the perception and belief variables. Perceptions and beliefs were measured on a five-point Likert scale. All statistical analyses were carried out using Stata Software Version $16 .^{25}$

\section{Results}

\section{Demographic Information}

There were 343 responses ( $76 \%$ female) from 850 employees at the Queen Elizabeth Hospital (response rate $=40.4 \%)($ Table 1$)$. The majority $(58 \%)$ were over age 35 , and males were significantly older than females $(p=0.008)$ (Table 1$)$. Approximately $34 \%$ of those surveyed were married, and men $(55.5 \%)$ were more likely to be married than women $(28.1 \%)$. Most respondents were of Barbadian nationality (83.7\%). Nurses were the largest occupational group (42\%). The majority of respondents did not report a chronic illness $(74.1 \%)$.

\section{Knowledge of COVID-I9 Vaccines}

Most respondents (61.5\%) reported that their knowledge of COVID-19 vaccines was good. Men were more likely than women to be of this opinion (Table 1).

\section{Vaccine Intent and Hesitancy}

Of the 343 respondents, $55.1 \%$ indicated they would accept the COVID-19 vaccine if it were available, $23.9 \%$ preferred to see how it affected others, $14.0 \%$ did not intend to get it soon, and $7.0 \%$ stated that they would never get the vaccine. In total, $44.9 \%$ were labeled as vaccine hesitant. Nurses indicated less willingness to receive the vaccine $(50.7 \%)$ in comparison to doctors (59.7\%) and other HCPs (56.2\%). More persons indicated an intention to take the COVID-19 vaccine (55.1\%) than indicated intention to take the seasonal influenza vaccine $(21.0 \%)$ in 2021.

We explored whether perceived knowledge and selected socio-demographic factors affected reported intent to take the vaccine (Table 2). After bivariate analyses, age, sex, nationality, whether you had taken the flu vaccine in 2020, and perceived knowledge of COVID-19 were all statistically significantly associated with reported intent

Table I Socio-Demographic Characteristics, Vaccine Behavior, and Perceived Knowledge by Gender

\begin{tabular}{|c|c|c|c|c|}
\hline Characteristics & $\begin{array}{c}\text { Total }(n=343) ; \% \\
(n)\end{array}$ & $\begin{array}{c}\text { Male }(n=83) ; \%, \\
(n)\end{array}$ & $\begin{array}{c}\text { Female }(n=260) ; \%, \\
(n)\end{array}$ & $\begin{array}{l}\text { Differences by } \\
\text { Gender }\end{array}$ \\
\hline \multicolumn{5}{|l|}{ Age Group } \\
\hline$\cdot 18-34$ & $42.0(144)$ & $33.7(28)$ & $44.6(116)$ & \\
\hline - $35+$ & $58.0(199)$ & $66.3(55)$ & $55.4 \mid 44)$ & $\mathrm{P}=0.080$ \\
\hline Married & 34.7 (119) & $55.5(46)$ & $28.1(73)$ & $P<0.000$ I \\
\hline Nationality: Barbadian & $83.7(287)$ & $80.7(67)$ & $84.6(220)$ & $P=0.4$ \\
\hline Chronic illness & $26.0(89)$ & $28.9(24)$ & $25.0(65)$ & $P=0.479$ \\
\hline \multicolumn{5}{|l|}{ Occupation } \\
\hline - Doctor & 34.7 (119) & $39.8(33)$ & $33.1(86)$ & \\
\hline - Nurse & $42.0(144)$ & $42.2(35)$ & $41.9(109)$ & $P=0.55 I$ \\
\hline - Allied health/Admin & $23.3(80)$ & $18.0(15)$ & $25.0(65)$ & \\
\hline Received flu vaccine in 2020. & $21.3(73)$ & $30.1(25)$ & I8.5 (48) & $\mathrm{P}=0.024$ \\
\hline I intend to take flu vaccine in 2021 . & $39.4(135)$ & $54.2(45)$ & $34.6(90)$ & $P=0.001$ \\
\hline $\begin{array}{l}\text { I intend to take COVID-19 vaccine in } \\
2021 .\end{array}$ & $55.1(189)$ & $67.5(56)$ & $51.2(133)$ & $P=0.009$ \\
\hline \multicolumn{5}{|l|}{ Perceived knowledge } \\
\hline - Good & $61.5(2 \mathrm{II})$ & $74.7(62)$ & $57.3(149)$ & \\
\hline - Average & $37.0(127)$ & $24.1(20)$ & $41.2(107)$ & $P=0.018$ \\
\hline - Poor & I.5 (5) & $1.2(I)$ & I.5 (4) & \\
\hline
\end{tabular}


Table 2 Adjusted and Unadjusted Predictors of Vaccine Intent

\begin{tabular}{|c|c|c|c|}
\hline $\begin{array}{l}\text { Variables in the Multivariable } \\
\text { Logistic Model }\end{array}$ & $\begin{array}{l}\text { Proportion Who Intends to Take COVID-19 } \\
\text { Vaccine, \% }\end{array}$ & $\begin{array}{l}\text { Unadjusted OR } \\
\qquad(95 \% \mathrm{Cl})\end{array}$ & $\begin{array}{c}\text { Multivariable } \\
\text { Adjusted } \\
\text { OR }(95 \% \mathrm{Cl})\end{array}$ \\
\hline \multicolumn{4}{|l|}{ Age } \\
\hline $18-34$ & 47.2 & 1.00 & 1.00 \\
\hline 35 plus & 60.8 & $1.73(1.12,2.67)$ & $1.21(0.73,2.00)$ \\
\hline \multicolumn{4}{|l|}{ Sex } \\
\hline Female & 51.2 & 1.00 & 1.00 \\
\hline Male & 67.5 & $1.98(1.18,3.33)$ & $\mathrm{I} .47(0.83,2.6 \mathrm{I})$ \\
\hline \multicolumn{4}{|l|}{ Marital status } \\
\hline Single & 48.7 & 1.00 & 1.00 \\
\hline Married & 67.2 & $2.16(1.36,3.44)$ & $1.38(0.80,2.37)$ \\
\hline \multicolumn{4}{|l|}{ Nationality } \\
\hline Barbadian & 49.8 & 1.00 & 1.00 \\
\hline Non-Barbadian & 82.1 & $4.63(2.25,9.53)$ & $4.34(2.04,9.27)^{*}$ \\
\hline \multicolumn{4}{|l|}{ Chronic illness } \\
\hline No & 53.9 & 1.00 & 1.00 \\
\hline Yes & 58.4 & $1.20(0.74,1.96)$ & I.2I (0.7I, 2.09) \\
\hline \multicolumn{4}{|l|}{ Occupation } \\
\hline Nurses & 50.7 & 1.00 & 1.00 \\
\hline Allied Health & 56.2 & $1.25(0.72,2.17)$ & $1.78(0.98,3.24)$ \\
\hline Doctors & 59.7 & $1.44(0.88,2.35)$ & $1.50(0.88,2.57)$ \\
\hline \multicolumn{4}{|l|}{ Flu vaccine in 2020} \\
\hline No & 49.3 & 1.00 & 1.00 \\
\hline Yes & 76.7 & $3.39(1.88,6.13)$ & $3.06(1.64,5.73)^{*}$ \\
\hline \multicolumn{4}{|l|}{ Perceived knowledge } \\
\hline Average/Poor & 43.9 & 1.00 & 1.00 \\
\hline Good & 62.1 & $2.09(1.34,3.25)$ & $1.58(0.97,2.57)$ \\
\hline
\end{tabular}

Notes: $* 95 \% \mathrm{Cl}$ does not include I indicating a statistically significant association between vaccine intent and nationality and flu vaccine acceptance in 2020 .

to take the COVID-19 vaccine. Persons over age 35 years had higher odds of expressing intent to take the COVID19 vaccine compared to those 18-34; OR 1.73 [95\% CI $(1.12,2.67)]$. Similarly, males were more likely to express intent to take the vaccine; OR 1.98 [95\% CI $(1.18,3.33)]$. After adjustment in the multivariable logistic regression model, only nationality and having received the 2020 seasonal flu vaccine were statistically significant predictors of intent to take the COVID-19 vaccine.

\section{Vaccine Literacy}

We used six items from a previously developed eight-item self-report questionnaire to measure vaccine literacy. ${ }^{22}$ Our adaptation demonstrated adequate internal consistency (Cronbach $\alpha=0.79$ ). We compared mean and median vaccine literacy scores by socio-demographic factors (age group, gender, marital status, nationality, occupational status), vaccine behavior, and perceived vaccine knowledge (Table 3). The mean vaccine literacy score differed significantly for three groups: Married persons, those who reported intent to take the COVID-19 vaccine, and those with perceived higher knowledge of the vaccine all had higher vaccine literacy scores (Table 3). Higher perceived knowledge was associated with a higher vaccine literacy score [3.48 (95\% CI 3.42, 3.54)] compared to those who reported that their knowledge was average or poor $[3.16(95 \%$ CI $3.08,3.24)]$, and persons who positively indicated that they would take the vaccine had higher vaccine literacy scores [3.46 (95\% CI 3.40, 3.52)] than those who indicated they were not ready or would not take the vaccine $[3.23(95 \%$ CI $3.15,3.30)]$. 
Table 3 Vaccine Literacy Scores by Demographic Variables, Vaccine Taking Behavior, and Perceived COVID-19 Vaccine Knowledge

\begin{tabular}{|c|c|c|}
\hline & $\begin{array}{c}\text { Vaccine Literacy, } \\
\text { Mean }(95 \% \mathrm{Cl})\end{array}$ & $\begin{array}{c}\text { Vaccine Literacy, } \\
\text { Median (IQR) }\end{array}$ \\
\hline \multicolumn{3}{|l|}{ Age Group } \\
\hline - 18-34 & $3.33(3.26,3.40)$ & $3.00(3.33,3.67)$ \\
\hline - $35+$ & $3.38(3.31,3.44)$ & $3.00(3.33,3.83)$ \\
\hline \multicolumn{3}{|l|}{ Married** } \\
\hline •Yes & $3.48(3.40,3.56)$ & $3.00(3.33,3.67)$ \\
\hline - No & $3.29(3.23,3.36)$ & $3.50(3.17,3.83)$ \\
\hline \multicolumn{3}{|l|}{ Sex } \\
\hline - Female & $3.32(3.27,3.38)$ & $3.00(3.33,3.67)$ \\
\hline - Male & $3.46(3.36,3.56)$ & $3.50(3.17,3.83)$ \\
\hline \multicolumn{3}{|l|}{ Nationality } \\
\hline - Barbadian & $3.34(3.28,3.39)$ & $3.33(3.00,3.67)$ \\
\hline - Others & $3.48(3.36,3.59)$ & $3.50(3.17,3.83)$ \\
\hline \multicolumn{3}{|l|}{ Chronic illness \%, (n) } \\
\hline - Yes & $3.40(3.30,3.49)$ & $3.50(3.00,3.67)$ \\
\hline - No & $3.34(3.29,3.40)$ & $3.00(3.33,3.67)$ \\
\hline \multicolumn{3}{|l|}{ Occupation } \\
\hline - Doctor & $3.43(3.36,3.5 \mathrm{I})$ & $3.50(3.17,3.67)$ \\
\hline - Nurse & $3.29(3.21,3.38)$ & $3.33(3.00,3.67)$ \\
\hline - Allied health/ & $3.36(3.27,3.46)$ & $3.33(3.08,3.67)$ \\
\hline Admin & & \\
\hline \multicolumn{3}{|l|}{ Received flu vaccine in } \\
\hline \multicolumn{3}{|l|}{ 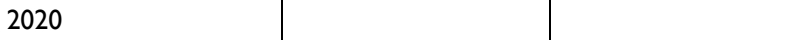 } \\
\hline - Yes & $3.43(3.31,3.55)$ & $3.50(3.00,3.83)$ \\
\hline - No & $3.34(3.28,3.39)$ & $3.33(3.00,3.67)$ \\
\hline \multicolumn{3}{|l|}{ I intend to take the } \\
\hline \multicolumn{3}{|l|}{ COVID-19 vaccine ${ }^{* *}$} \\
\hline - Yes & $3.46(3.40,3.52)$ & $3.50(3.17,3.83)$ \\
\hline - Later/No & $3.23(3.15,3.30)$ & $3.17(3.00,3.50)$ \\
\hline \multicolumn{3}{|l|}{ Perceived } \\
\hline \multicolumn{3}{|l|}{ knowledge** } \\
\hline - Good & $3.48(3.42,3.54)$ & $3.50(3.17,3.83)$ \\
\hline - Average/Poor & $3.16(3.08,3.24)$ & $3.17(3.00,3.50)$ \\
\hline
\end{tabular}

Notes: **These were statistically significant at the $5 \%$ level of testing. Note the $95 \%$ $\mathrm{Cl}$ around the means do not overlap with each other for the stated categories.

We further explored the correlations between vaccine literacy scores and questions about perceptions and beliefs (Table 4). We found significantly higher literacy scores among those who believed that (1) vaccine producers care for their well-being; (2) COVID-19 vaccination should be compulsory once available, and (3) COVID-19 vaccines are safe. Vaccine literacy scores were also significantly higher among those who were willing to pay $[3.50(95 \%$ CI $3.42,3.57)]$ versus those who were not $[3.28$ (95\% CI 3.22, 3.35)]; and those who would recommend the vaccine to a friend [3.46 (95\% CI 3.41, $3.51)]$ versus those who would not [3.11 $(95 \%$ CI 3.02 , 3.21)] (Table 4).

\section{Discussion}

In this study, we evaluated vaccine acceptance/hesitancy towards COVID-19 vaccination among HCPs in Barbados. The main findings of our study revealed that more than half of HCPs $(55.1 \%)$ stated that they would get vaccinated if a COVID-19 vaccine were available, and almost one-quarter (24\%) intended to get vaccinated after reviewing how vaccines affected others. Overall vaccine hesitancy among HCPs in the QEH was $44.9 \%$, which is consistent with findings of a study in the Cayman Islands $(48 \%),{ }^{26}$ but much higher than a larger regional study (23\%) conducted among HCPs in 14 Caribbean countries. $^{12}$

Previous studies reported COVID-19 vaccine hesitancy ranging from $32 \%$ to $62 \%$ among adult populations of several Caribbean countries. ${ }^{27}$ A study conducted during December 2020-January 2021 found a high COVID-19 vaccine hesitancy rate $(62.3 \%)$ among the adult population in Trinidad and Tobago. ${ }^{28}$ Another, conducted by Johns Hopkins University during September 2020, reported that $59 \%$ of respondents in Trinidad and Tobago and $72 \%$ of Jamaicans were vaccine-hesitant. These findings are comparable to the findings of the Institute of Global Health Innovation, Imperial College in 15 countries between November 2020 and mid-January 2021, which found that $54 \%$ of respondents indicated an intention to receive a COVID-19 vaccine if available (vaccine hesitancy rate: $46 \%)^{29}$

Vaccine hesitancy poses a major threat to the success of vaccination in preventing disease and death from COVID-19. Hesitancy among HCPs, documented in a number of recent studies, is particularly concerning. Studies conducted globally on COVID-19 vaccine acceptance among HCPs have reported wide variations in vaccine hesitancy ranging from $8.3 \%$ to $72.4 \%{ }^{4,8,14,15,30-39}$ Notably, some studies were conducted before vaccines were approved and/or rolled out, ${ }^{15}$ and HCPs in some studies had already received vaccines. ${ }^{32}$ Our study was conducted at the start of the national vaccine campaign in Barbados began in February 2021. The vaccine hesitancy we report is consistent with some studies conducted 
Table 4 Correlations Between Vaccine Literacy (VL) Scores and Perceptions and Beliefs About COVID-19 Vaccines

\begin{tabular}{|c|c|c|c|c|}
\hline \multirow[t]{2}{*}{ Perceptions and Beliefs About COVID-19 Vaccines } & \multirow[t]{2}{*}{ Agree (n, \%) } & \multicolumn{2}{|c|}{ VL Interactive Literacy Score } & \multirow{2}{*}{$\begin{array}{c}\text { Spearman } \\
\text { Correlation with } \\
\text { VL Score }\end{array}$} \\
\hline & & $\begin{array}{l}\text { Agree Mean } \\
(95 \% \mathrm{Cl})\end{array}$ & $\begin{array}{c}\text { Not Agree } \\
\text { Mean }(95 \% \mathrm{Cl})\end{array}$ & \\
\hline The vaccine producers care for my well-being. & $149,43.4 \%$ & $3.49(3.42,3.56)$ & $3.26(3.18,3.32)$ & 0.23 \\
\hline $\begin{array}{l}\text { The local authorities should check the efficacy of the vaccines } \\
\text { prior to implementation. }\end{array}$ & $294,85.7 \%$ & $3.39(3.34,3.44)$ & $3.19(3.03,3.35)$ & 0.13 \\
\hline COVID-19 vaccination should be compulsory, once available. & $101,29.5 \%$ & $3.51(3.43,3.60)$ & $3.29(3.23,3.35)$ & 0.13 \\
\hline $\begin{array}{l}\text { The health authorities will succeed in vaccinating the entire } \\
\text { population }\end{array}$ & $88,25.7 \%$ & $3.46(3.37,3.55)$ & $3.32(3.26,3.38)$ & 0.02 \\
\hline I will pay a fee to be vaccinated & $121,35.3 \%$ & $3.50(3.42,3.57)$ & $3.28(3.22,3.35)$ & 0.16 \\
\hline $\begin{array}{l}\text { I will recommend vaccines for others (eg, relatives, patients } \\
\text { etc.) }\end{array}$ & $239,69.7 \%$ & $3.46(3.4 I, 3.5 I)$ & $3.11(3.02,3.21)$ & 0.37 \\
\hline
\end{tabular}

after the COVID-19 vaccine received approval, for example, in Bangladesh $(56.2 \%){ }^{15}$ the US $\left(44.7 \%{ }^{37}\right.$ and $42.5 \%{ }^{36}$ ), and Germany (43\%). ${ }^{40}$ A UK study, conducted in February 2021 to assess the actual uptake of vaccines, reported that $64.5 \%$ of healthcare workers in an NHS Trust had received COVID-19 vaccination. ${ }^{41}$ However, our findings diverge from those of German (February 2021), US (December 2020), and Canadian (December 2020) studies that reported low vaccine hesitancy rates of $8.3 \%,{ }^{32}$ $14 \%,{ }^{31}$ and $19.1 \%,{ }^{30}$ respectively. A meta-analytic paper reviewed studies conducted from June to October 2020 with large nationally representative samples $(n \geq 1000$ each) from 13 countries $(n=58,656)$ and found that vaccine acceptance decreased and vaccination hesitancy increased as the pandemic progressed. ${ }^{42}$ Holzmann-Littig et $\mathrm{al}^{32}$ note that vaccine hesitancy in healthcare workers is multifactorial and, therefore, that comparisons should be done cautiously as acceptance was defined differently, measured on different scales, using different questionnaires with different numbers of items asked in different countries and time periods.

Moreover, vaccine acceptance may change during the pandemic because of vaccine campaigns and peer influence from those who were already vaccinated. ${ }^{32}$ The phenomenon of vaccine hesitancy among healthcare providers needs special attention, as HCPs function as role models to "influence local vaccination rates in individuals and at a population level" 43 and because of their greater personal exposure to COVID-19 infections. ${ }^{43,44}$ Ultimately, poor vaccination acceptance undermines efforts to reach the herd immunity threshold for a given population. ${ }^{45}$
After adjustment in the multivariable logistic regression model, we found that receiving the seasonal flu vaccine in 2020 was a statistically significant predictor of reported intent to take the COVID-19 vaccine. Several previous studies reported that last year's flu vaccination behavior predicted COVID-19 vaccine acceptance. ${ }^{38,46,47}$ The flu vaccine coverage in our study was $21.3 \%$, which is lower than in some other surveys of healthcare workers. ${ }^{32,48}$ This may be due to the low self-perceived risk to health of contracting influenza among HCPs. ${ }^{49}$ Our findings mirror those of Gagneux-Brunon et $\mathrm{al}^{33}{ }^{33}$ who reported a significant positive association of selfperceived risk of COVID-19 and prior flu vaccination with willingness to get the vaccine among French HCPs. Importantly, low uptake of the flu vaccine may cause increased absenteeism due to illness, ${ }^{50}$ putting a strain on the remaining staff to carry out clinical services, especially given the demands during the pandemic. ${ }^{51}$ HCPs should be encouraged to get both COVID-19 and influenza vaccines, ${ }^{33}$ as coinfections may cause severe symptoms and serious outcomes. ${ }^{50}$ It is encouraging that more than half of HCPs (55.1\%) in our study showed interest in taking the flu vaccine in 2021.

Health literacy and perceived knowledge are especially important in the context of the pandemic, as contradictory and fake news reports related to COVID-19 are common in print, broadcast, and, especially, social media. The surge of misinformation, mainly in the online community, has negative impact on the vaccination programs and has contributed to increased vaccine hesitancy among the general population. ${ }^{52,53}$ We found that higher perceived 
knowledge was associated with higher vaccine literacy scores, and persons who indicated that they would take the vaccine had a higher vaccine literacy score. This is consistent with previous findings that COVID-19 vaccine knowledge was significantly associated with vaccination acceptance. ${ }^{32}$ Prior studies have shown that nurses with higher vaccine literacy were more likely to advise their patients to take the influenza ${ }^{54}$ and Human Papillomavirus $(\mathrm{HPV})^{55}$ vaccines. Health literacy helps people to recognize the reasons behind medical recommendations and consider the outcomes of their possible actions, even as vaccine hesitancy is promoted by the infodemic of misinformation and disinformation related to COVID-19. ${ }^{56,57}$ Hence, there is a need to proactively counter false claims and disseminate evidence-based information to promote vaccine literacy. ${ }^{8,38}$

We also found that HCPs who had higher vaccine literacy scores believed that COVID-19 vaccines were safe and would recommend the vaccine to a friend. Past studies have shown that the most important independent predictor of vaccine hesitancy among HCPs is fear of compromised quality resulting from expedited COVID19 vaccine development. ${ }^{34,38}$ Perceived vaccine safety was also significantly associated with increased probability of choosing vaccination in previous surveys of the general population. ${ }^{35}$ As vaccine hesitancy an obstacle to ending this pandemic, public trust in the vaccination program must be continually strengthened by the government, HCPs, and other stakeholders. ${ }^{53}$ Physicians should be actively engaging their communities to educate the public about efficacy and safety of the available vaccines and promote transparency in government policies and programs related to vaccination. Providing relevant and current information is essential, as is vaccine advocacy by trusted colleagues, to address vaccine hesitancy among HCPs. ${ }^{30,43}$

We recognize that the actions of HCPs function among other powerful influences on vaccine success. Specific ingredients of effective public health messaging are not the same in all communities, and generic public information efforts may not appreciate relevant contextual factors. Collaborative approaches that consider and incorporate cultural and faith-based influences and actors are needed to increase vaccine acceptance rates ${ }^{52}$ and support comprehensive public health response. We further appreciate the fundamental importance of personal responsibility, advocated in a recent communication by Capulong, ${ }^{53}$ for citizens to engage in mindful critical appraisal of public health information and to educate others. Neither HCPs nor governments operate in isolation.

\section{Strengths/Limitations of the Study}

A significant strength of this study is that the survey was conducted at the onset of the national vaccine rollout campaign but after the COVID-19 vaccines had been approved in Barbados. Understanding the perspectives of HCPs is important because their roles as trusted sources of health information and as advocates during vaccination influence rollout campaigns. Significant limitations include a relatively low response rate $(40 \%)$ and data collection at a single tertiary hospital, which may impact generalizability, as the study did not include primary care health workers (selection bias). This survey was conducted during the initial stage of the vaccination program in Barbados; respondents' beliefs at the time of the survey may not reflect current beliefs and may have changed as the vaccine campaign has progressed and expanded. As this is a self-reported survey, the responses partly depended on the respondents' sincerity and technological literacy, and HCPs who were unvaccinated may have been less likely to complete the questionnaire.

\section{Conclusions}

This study examined vaccine hesitancy among HCPs just before the initiation of COVID-19 vaccine administration at the Queen Elizabeth Hospital, Barbados. We found that more than four out of ten HCPs were vaccine hesitant. Public health measures such as social distancing and quarantine may not be sufficient in themselves to halt the spread of COVID-19. Therefore, vaccination is critically important for reducing the prevalence of severe symptoms of COVID-19 infection, as well as achieving herd immunity in Barbados and elsewhere. Vaccination of frontline HCPs will help ensure an adequate workforce is available to treat patients.

Further, perceptions of HCPs have important implications for vaccine uptake in Barbados generally. High vaccine hesitancy among HCPs in Barbados presents and urgent need for appropriate strategies to provide updated and clear messages through trusted channels regarding the safety, efficacy, risks, and benefits of currently available COVID-19 vaccines. Implementation of continuing education for HCPs would be useful to address vaccine misinformation, which has implications for both HCP vaccine uptake and recommendations HCPs provide to patients. 


\section{Data Sharing Statement}

The datasets of the current study are available from the corresponding author on reasonable request.

\section{Ethics Approval and Consent to Participate}

This study was approved by the University of the West Indies, Cave Hill Campus/Barbados Ministry of Health and Wellness Research Ethics Committee/Institutional Review Board (IRB No. 210101-B) and the QEH Ethics Committee (Ref: 12021). All participants provided informed consent in accordance with the Declaration of Helsinki.

\section{Acknowledgments}

The authors would like to thank the HCPs of QEH who completed the questionnaire.

\section{Disclosure}

Dr Md Anwarul Azim Majumder is the Editor-in-Chief of the journal Advances in Medical Education and Practice and reports no other potential conflicts of interest for this work. The other authors report no conflicts of interest in this work.

\section{References}

1. Detoc M, Bruel S, Frappe P, Tardy B, Botelho-Nevers E, GagneuxBrunon A. Intention to participate in a COVID-19 vaccine clinical trial and to get vaccinated against COVID-19 in France during the pandemic. Vaccine. 2020;38(45):7002-7006. doi:10.1016/j. vaccine. 2020.09.041

2. Habas K, Nganwuchu C, Shahzad F, et al. Resolution of coronavirus disease 2019 (COVID-19). Expert Rev Anti Infect Ther. 2020;18 (12):1201-1211. doi:10.1080/14787210.2020.1797487

3. Rahman S, Montero MTV, Rowe K, Kirton R, Kunik F Jr. Epidemiology, pathogenesis, clinical presentations, diagnosis and treatment of COVID-19: a review of current evidence. Expert Rev Clin Pharmacol. 2021;14(5):601-621. doi:10.1080/17512433.2021.1902303

4. Shekhar R, Sheikh AB, Upadhyay S, et al. COVID-19 vaccine acceptance among health care workers in the United States. Vaccines (Basel). 2021;9(2):Feb. doi:10.3390/vaccines9020119

5. Tyson A, Johnson C, F C. U.S. Public Now Divided Over Whether to Get COVID-19 Vaccine. Pew Research Center; 2020. Available from: https://www.pewresearch.org/science/2020/09/17/u-s-public-nowdivided-over-whether-to-get-covid-19-vaccine/. Accessed November 19, 2021.

6. Garrett L. COVID-19: the medium is the message. Lancet. 2020;395 (10228):942-943. doi:10.1016/s0140-6736(20)30600-0

7. Larson HJ, Smith DM, Paterson P, et al. Measuring vaccine confidence: analysis of data obtained by a media surveillance system used to analyse public concerns about vaccines. Lancet Infect Dis. 2013;13 (7):606-613. doi:10.1016/s1473-3099(13)70108-7

8. Gadoth A, Halbrook M, Martin-Blais R, et al. Cross-sectional assessment of COVID-19 vaccine acceptance among healthcare workers in Los Angeles. medRxiv. doi:10.1101/2020.11.18.20234468
9. MacDonald NE. Vaccine hesitancy: definition, scope and determinants. Vaccine. 2015;33(34):4161-4164. doi:10.1016/j. vaccine. 2015.04.036

10. Phadke VK, Bednarczyk RA, Salmon DA, Omer SB. Association between vaccine refusal and vaccine-preventable diseases in the United States: a review of measles and pertussis. JAMA. 2016;315 (11):1149-1158. doi:10.1001/jama.2016.1353

11. Sallam M. COVID-19 vaccine hesitancy worldwide: a concise systematic review of vaccine acceptance rates. Vaccines (Basel). 2021;9 (2):Feb. doi:10.3390/vaccines 9020160

12. Pan American Health Organization (PAHO). Concerns, Attitudes, and Intended Practices of Healthcare Workers to COVID-19 Vaccination in the Caribbean; 2021.

13. Cascini F, Pantovic A, Al-Ajlouni Y, Failla G, Ricciardi W. Attitudes, acceptance and hesitancy among the general population worldwide to receive the COVID-19 vaccines and their contributing factors: a systematic review. EClinicalMedicine. 2021;40:101113. doi:10.1016/j.eclinm.2021.101113

14. Kabamba Nzaji M, Kabamba Ngombe L, Ngoie Mwamba G, et al. Acceptability of vaccination against COVID-19 among healthcare workers in the democratic Republic of the Congo. Pragmat Obs Res. 2020;11:103-109. doi:10.2147/por.s271096

15. Alam A, Majumder MAA, Haque $M$, et al. Disproportionate COVID-19 vaccine acceptance rate among healthcare professionals on the eve of nationwide vaccine distribution in Bangladesh. Expert Rev Vaccines. 2021. doi:10.1080/14760584.2021.1951248

16. Ashok N, Krishnamurthy K, Singh K, Rahman S, Majumder MAA. High COVID-19 vaccine hesitancy among healthcare workers: should such a trend require closer attention by policymakers? Cureus. 2021;13(9):e17990. doi:10.7759/cureus. 17990

17. Covid Observer. Coronavirus in Barbados. Available from: https:// covid.observer/bb/. Accessed November 1, 2021

18. Worldometer. Barbados. Available from: https://www.worldometers. info/coronavirus/country/barbados/. Accessed November 1, 2021.

19. The Barbados Government Information Service (BGIS). COVID-19 Update For Sunday, October 31. Available from: https://gisbarbados. gov.bb/blog/covid-19-update-for-sunday-october-31/. Accessed November 1, 2021.

20. Muqattash R, Niankara I, Traoret RI. Survey data for COVID-19 vaccine preference analysis in the United Arab Emirates. Data Brief. 2020;33:106446. doi:10.1016/j.dib.2020.106446

21. Wang J, Jing R, Lai X, et al. Acceptance of COVID-19 vaccination during the COVID-19 Pandemic in China. Vaccines (Basel). 2020;8 (3). doi:10.3390/vaccines 8030482

22. Biasio LR, Bonaccorsi G, Lorini C, Pecorelli S. Assessing COVID-19 vaccine literacy: a preliminary online survey. Hum Vaccin Immunother. 2021;17(5):1304-1312. doi:10.1080/ 21645515.2020.1829315

23. Shed. HLT. Health Literacy about Vaccination of Adults Italian (HLVa-IT). Available from: https://healthliteracy.bu.edu/hlva-it. Accessed July 13, 2021.

24. D Nutbeam. Health literacy as a public health goal: a challenge for contemporary health education and communication strategies into the 21st century. Health Promot Int. 2020;15(3):259-267.

25. StataCorp. Stata Statistical Software: Release 16. StataCorp LLC; 2019.

26. Cayman Islands Health Services Authority. Health Care Industry COVID-19 Vaccine Survey; 2020.

27. CARICOM. CARPHA launches COVID-19 vaccine acceptance survey; 2021. Available from: https://caricom.org/carpha-launchescovid-19-vaccine-acceptance-survey/. Accessed July 5, 2021.

28. Sa B, Dwarica D, Leotaud T, et al. Acceptance and Attitudes towards COVID-19 Vaccine among the Adult Population in Trinidad And Tobago. presented at: The COVID-19 Vaccine Preparedness Virtual Symposium- The Healthcare Worker Edition; 7th February 2021; St. Augustine, Trinidad and Tobago. 
29. Institute, of Global Health Innovation. Global attitudes towards a COVID-19 vaccine; 2021. Available from: https://www.imperial.ac. uk/media/imperial-college/institute-of-globalhealth-innovation /GlobalVaccineInsights_ICL-Covid-19-Behaviour-Tracker-

EMBARGOED-00.01-04.02.2021.pdf. Accessed November 19, 2021

30. Dzieciolowska S, Hamel D, Gadio S, et al. Covid-19 vaccine acceptance, hesitancy, and refusal among Canadian healthcare workers: a multicenter survey. Am J Infect Control. 2021. doi:10.1016/j. ajic.2021.04.079

31. Schrading WA, Trent SA, Paxton JH, et al. Vaccination rates and acceptance of SARS-CoV-2 vaccination among U.S. emergency department health care personnel. Acad Emerg Med. 2021;455-458(vol):4.

32. Holzmann-Littig C, Braunisch MC, Kranke P, et al. COVID-19 vaccination acceptance among healthcare workers in Germany. medRxiv. 2021. doi:10.1101/2021.04.20.21255794

33. Gagneux-Brunon A, Detoc M, Bruel S, et al. Intention to get vaccinations against COVID-19 in French healthcare workers during the first pandemic wave: a cross-sectional survey. $J$ Hosp Infect. 2021;108:168-173. doi:10.1016/j.jhin.2020.11.020

34. Barry M, Temsah M-H, Alhuzaimi A, et al. COVID-19 vaccine confidence and hesitancy among healthcare workers: a cross-sectional survey from a MERS-CoV experienced nation. medRxiv. 2020. doi:10.1101/2020.12.09.20246447

35. Fu C, Wei Z, Pei S, Li S, Sun X, Liu P. Acceptance and preference for COVID-19 vaccination in health-care workers (HCWs). medRxiv. 2020. doi:10.1101/2020.04.09.20060103

36. Shaw J, Stewart T, Anderson KB, et al. Assessment of U.S. health care personnel (HCP) attitudes towards COVID-19 vaccination in a large university health care system. Clin Infect Dis. 2021. doi:10.1093/cid/ciab054

37. Meyer MN, Gjorgjieva T, Rosica D. Trends in health care worker intentions to receive a COVID-19 vaccine and reasons for hesitancy. JAMA Netw Open. 2021;4(3):e215344. doi:10.1001/ jamanetworkopen.2021.5344

38. Verger P, Scronias D, Dauby N, et al. Attitudes of healthcare workers towards COVID-19 vaccination: a survey in France and French-speaking parts of Belgium and Canada, 2020. Euro Surveill. 2021;26(3). doi:10.2807/1560-7917.Es.2021.26.3.2002047

39. Dror AA, Eisenbach N, Taiber S, et al. Vaccine hesitancy: the next challenge in the fight against COVID-19. Eur J Epidemiol. 2020;775779(vol):8.

40. Nohl A, Afflerbach C, Lurz C, et al. Acceptance of COVID-19 vaccination among front-line health care workers: a nationwide survey of emergency medical services personnel from Germany. Vaccines (Basel). 2021;9(5). doi:10.3390/vaccines9050424

41. Martin CA, Marshall C, Patel P, et al. Association of demographic and occupational factors with SARS-CoV-2 vaccine uptake in a multi-ethnic UK healthcare workforce: a rapid real-world analysis. medRxiv. 2021. doi:10.1101/2021.02.11.21251548

42. Robinson E, Jones A, Lesser I, Daly M. International estimates of intended uptake and refusal of COVID-19 vaccines: a rapid systematic review and meta-analysis of large nationally representative samples. Vaccine. 2021;39(15):2024-2034. doi:10.1016/j. vaccine.2021.02.005

43. Razai MS, Chaudhry UAR, Doerholt K, Bauld L. Covid-19 vaccination hesitancy. BMJ. 2021;373:n1138.
44. Verger P, Dubé E. Restoring confidence in vaccines in the COVID-19 era. Expert Rev Vaccines. 2020;19(11):991-993. doi:10.1080/ 14760584.2020.1825945

45. Randolph HE, Barreiro LB. Herd immunity: understanding COVID-19. Immunity. 2020;52(5):737-741. doi:10.1016/j. immuni.2020.04.012

46. Wang K, Wong ELY, Ho KF, et al. Intention of nurses to accept coronavirus disease 2019 vaccination and change of intention to accept seasonal influenza vaccination during the coronavirus disease 2019 pandemic: a cross-sectional survey. Vaccine. 2020;38 (45):7049-7056. doi:10.1016/j.vaccine.2020.09.021

47. Kose S, Mandiracioglu A, Sahin S, Kaynar T, Karbus O, Ozbel Y. Vaccine hesitancy of the COVID-19 by health care personnel. Int J Clin Pract. 2021;75(5):e13917. doi:10.1111/ijcp.13917

48. Neufeind J, Wenchel R, Boedeker B, Wicker S, Wichmann O. Monitoring influenza vaccination coverage and acceptance among health-care workers in German hospitals - results from three seasons. Hum Vaccin Immunother. 2021;17(3):664-672. doi:10.1080/21645515.2020.1801072

49. Lorenc T, Marshall D, Wright K, Sutcliffe K, Sowden A. Seasonal influenza vaccination of healthcare workers: systematic review of qualitative evidence. BMC Health Serv Res. 2017;17(1):732. doi:10.1186/s12913-017-2703-4

50. Costantino C, Casuccio A, Caracci F, et al. Impact of Communicative and Informative Strategies on Influenza Vaccination Adherence and Absenteeism from Work of Health Care Professionals Working at the University Hospital of Palermo, Italy: a Quasi-Experimental Field Trial on Twelve Influenza Seasons. Vaccines (Basel). 2019;8(1). doi: $10.3390 /$ vaccines 8010005

51. Groenewold MR, Burrer SL, Ahmed F, Uzicanin A, Free H, Luckhaupt SE. Increases in health-related workplace absenteeism among workers in essential critical infrastructure occupations during the COVID-19 pandemic - United States, March-April 2020. MMWR Morb Mortal Wkly Rep. 2020;69(27):853-858. doi:10.15585/mmwr. mm6927a1

52. Gozum IE, Capulong HG, Gopez JM, Galang JR. Culture Religion and the state: towards a multidisciplinary approach to ensuring public health during the COVID-19 pandemic (and Beyond). Risk Manag Healthc Policy. 2021;14:3395-3401. doi:10.2147/rmhp.s318716

53. Capulong HGM. Mindfulness as key in easing COVID-19 vaccine hesitancy. $J$ Public Health (Oxf). 2021;43(2):e338-e339. doi:10.1093/pubmed/fdab050

54. Zhang J, While AE, Norman IJ. Nurses' vaccination against pandemic H1N1 influenza and their knowledge and other factors. Vaccine. 2012;30(32):4813-4819. doi:10.1016/j.vaccine.2012.05.012

55. Wamai RG, Ayissi CA, Oduwo GO, et al. Awareness, knowledge and beliefs about HPV, cervical cancer and HPV vaccines among nurses in Cameroon: an exploratory study. Int J Nurs Stud. 2013;50 (10):1399-1406. doi:10.1016/j.ijnurstu.2012.12.020

56. MacDonald NE. Fake news and science denier attacks on vaccines. What Can You Do? Can Commun Dis Rep. 2020;432-435:1112.

57. Farooq F, Rathore FA. COVID-19 vaccination and the challenge of infodemic and disinformation. J Korean Med Sci. 2021;36(10):e78. doi:10.3346/jkms.2021.36.e 78 


\section{Publish your work in this journal}

The Journal of Multidisciplinary Healthcare is an international, peerreviewed open-access journal that aims to represent and publish research in healthcare areas delivered by practitioners of different disciplines. This includes studies and reviews conducted by multidisciplinary teams as well as research which evaluates the results or conduct of such teams or healthcare processes in general. The journa

covers a very wide range of areas and welcomes submissions from practitioners at all levels, from all over the world. The manuscript management system is completely online and includes a very quick and fair peer-review system. Visit http://www.dovepress.com/testimonials. php to read real quotes from published authors. 